\title{
Three-dimensional coarsening dynamics of a conserved, nematic liquid crystal-isotropic fluid mixture
}

\author{
Rudimar L. Nós ${ }^{\mathrm{a}, *}$, Alexandre M. Roma ${ }^{\mathrm{b}}$, Carlos J. García-Cervera ${ }^{\mathrm{c}}$, Hector \\ D. Ceniceros ${ }^{\mathrm{d}}$ \\ ${ }^{a}$ Departamento Acadêmico de Matemática, Universidade Tecnológica Federal do Paraná, \\ CEP 80230-901, Curitiba-PR, Brazil \\ ${ }^{b}$ Departamento de Matemática Aplicada, Universidade de São Paulo, Caixa Postal \\ 66281, CEP 05311-970, São Paulo-SP, Brazil \\ ${ }^{c}$ Department of Mathematics, University of California, Santa Barbara, CA 93106, \\ United States and Basque Center for Applied Mathematics, Alameda de Mazarredo 14 \\ 48009 Bilbao, Bizkaia, Spain \\ ${ }^{d}$ Department of Mathematics, University of California, Santa Barbara, CA 93106, \\ United States
}

\begin{abstract}
We present a numerical investigation of the three-dimensional coarsening dynamics of a nematic liquid crystal-isotropic fluid mixture using a conserved phase field model. The model is a coupled system for a generalized CahnHilliard equation for the order parameter $\phi$, related to the volume fraction of the nematic component, and a simplified de Gennes-Prost evolution equation for the director field $\mathbf{n}$, which describes the mean orientation of the rigid rod-like, liquid crystal molecules. We find that, as in the two-dimensional system, the orientational distortion induced by interfacial anchoring has profound effects both on the morphology and the coarsening rate. However, we identify significant differences in the three-dimensional and two-dimensional coarsening processes. In particular, we find a remarkable, new 3-stage late coarsening process with markedly different coarsening rates in the three-
\end{abstract}

\footnotetext{
* Corresponding author

Email addresses: rudimarnos@utfpr.edu.br (Rudimar L. Nós), roma@ime.usp.br (Alexandre M. Roma), cgarcia@math.ucsb.edu (Carlos J. García-Cervera), hdc@math.ucsb.edu (Hector D. Ceniceros)

$U R L$ : paginapessoal .utfpr.edu.br/rudimarnos (Rudimar L. Nós), www.ime.usp.br/ ${ }^{\sim}$ roma (Alexandre M. Roma), www.math.ucsb.edu/ ${ }^{\sim}$ garcia (Carlos J. García-Cervera), www.math.ucsb.edu/ hdc (Hector D. Ceniceros)
} 
dimensional bicontinuous phase separation with homeotropic anchoring, unseen in the two-dimensional system.

Keywords: Canh-Hilliard equation, Model B, nematic liquid crystal, nucleation, planar and homeotropic anchoring, semi-implicit methods, Adaptive Mesh Refinements.

\section{Introduction}

Phase separation of binary mixtures is a fundamental process in materials processing. The important phenomenon is characterized by a fast transition into an ordered phase consisting of domains rich in either component and followed by a very slow coarsening process until a steady state is reached $[7,6]$.

Mixtures in which one of the components is a liquid crystal or a liquid crystalline polymer offer a significant potential for applications and have received increased attention $[28,34,20,27,9,8,26]$.

We focus here on the three-dimensional phase separation and coarsening dynamics of a binary mixture of a nematic liquid crystal and an isotropic fluid, like a polymer. We use a conserved phase field model (Model B in the nomenclature of of Hohenberg and Halperin [18]) which couples a generalized Cahn-Hilliard equation for the order parameter $\phi$, related to the volume fraction of the species, with a simplified de Gennes-Prost evolution equation [16] for the director field $\mathbf{n}$, which describes the mean orientation of the rigid rod-like, liquid crystal molecules. The same model, which stems from that considered in [35], has been used in the two-dimensional study of Mata et al. [26] and the current work is a follow-up report on our findings for the corresponding three-dimensional system. Similar phase field models have been used extensively in phase separation $[14,25,19,4,1,2,21,22,5,35,37,36$, $3,38,13,39,12,30,17,33]$.

We find that the global distortion of the orientational field in the nematicrich phase, induced by strong interfacial anchoring, has a profound effect on the morphology and coarsening rate, just as it happens in 2D. Specifically, the steady-state morphology of the system can be largely controlled by the type of interfacial anchoring and the coarsening rate is significantly affected by anchoring-induced long-range orientational distortion. However, we observe substantial differences between the three-dimensional and two-dimensional coarsening dynamics. In particular, we identify a remarkable, new 3-stage late coarsening process with markedly different coarsening rates in the three- 
dimensional bicontinuous phase separation with homeotropic anchoring (n perpendicular to the surface), unseen in the two-dimensional system. We also obtain a notable minimal surface (a Schwarz P Surface) for one instance of $3 \mathrm{D}$ phase separation with homeotropic anchoring conditions.

The rest of the paper is organized as follows. In Section 2 we provide a description of the phase field model and the numerical methodology employed is described in Section 3. A summary of our numerical results is given in Section 4 and some concluding remarks are given in Section 5. Finally, data of an accuracy and convergence test of the numerical method are provided in the Appendix.

\section{Mathematical Model}

We focus on a system consisting of a conserved mixture of a nematic liquid crystal and an isotropic fluid, which undergoes phase separation in three dimensional space. The model is the same as that used in [26] except that here our domain is three-dimensional. The system can be described with an order parameter $\phi$ related to the species concentration $((1+\phi) / 2$ represents the nematic liquid crystal concentration and $(1-\phi) / 2$ the isotropic fluid concentration) and with the director field $\mathbf{n}$, which is a measure of the mean molecular orientation in the nematic liquid crystal phase. The pure, bulk phases are identified with $\phi=1$ and $\phi=-1$ for the nematic liquid crystal and the isotropic fluid, respectively. A narrow neighborhood of the level set $\phi=0$ provides a diffuse interface between the two species.

The free energy density of the system has three parts: a mixing energy $f_{\text {mix }}$, a bulk, orientational distortion energy of the nematic, $f_{\text {bulk }}$, and the anchoring energy related to the preferential orientation of the liquid crystal molecules at interfaces, $f_{\text {anch }}[35]$ :

$$
f(\phi, \mathbf{n}, \nabla \phi, \nabla \mathbf{n})=f_{\text {mix }}+\frac{1+\phi}{2} f_{\text {bulk }}+f_{\text {anch }},
$$


where

$$
\begin{aligned}
& f_{\text {mix }}= \frac{\lambda}{2}\left[|\nabla \phi|^{2}+\frac{\left(\phi^{2}-1\right)^{2}}{2 \varepsilon^{2}}\right], \\
& f_{\text {bulk }}= \frac{K}{2}\left[\nabla \mathbf{n}:(\nabla \mathbf{n})^{T}+\frac{\left(|\mathbf{n}|^{2}-1\right)^{2}}{2 \delta^{2}}\right], \\
& f_{\text {anch }}= \begin{cases}\frac{A}{2}(\mathbf{n} \cdot \nabla \phi)^{2} & \text { (planar anchoring), } \\
\frac{A}{2}\left[|\mathbf{n}|^{2}|\nabla \phi|^{2}-(\mathbf{n} \cdot \nabla \phi)^{2}\right] & \text { (homeotropic anchoring). }\end{cases}
\end{aligned}
$$

The parameter $\lambda$ in (2) is the strength of the mixing energy density and $\varepsilon$ is the capillary width. Equation (3) is the regularized Frank energy in which the elastic constants for splay, twist, and bend are all equal to $K$ and $(|\mathbf{n}|-$ $1)^{2} /\left(2 \delta^{2}\right)$ is a penalty term to approximately enforce the constraint $|\mathbf{n}|=1$. Finally, in (4), $A$ is the volumetric anchoring strength, which is related to the surface anchoring strength $W$ by $\varepsilon W=(2 \sqrt{2} / 3) A$ [35]. The specific choice of $f_{\text {anch }}$ for planar (homeotropic) anchoring in (4) favors alignment of the director field $\mathbf{n}$ tangential (normal) to nematic-isotropic fluid interfaces.

We consider a domain $\Omega=[0, L] \times[0, L] \times[0, L]$. The total free energy is

$$
F=\int_{\Omega} f(\phi, \mathbf{n}, \nabla \phi, \nabla \mathbf{n} .) d x
$$

The evolution of the order parameter is governed by the Cahn-Hilliard equation $[10,11]$

$$
\frac{\partial \phi}{\partial t}=\nabla \cdot[\gamma \nabla \mu]
$$

where $\gamma$ is the mobility, which in this work is taken to be constant, and

$$
\mu=\frac{\delta F}{\delta \phi} .
$$

Using (2)-(4) we obtain

$$
\mu=\lambda\left[-\nabla^{2} \phi+\frac{\phi\left(\phi^{2}-1\right)}{\varepsilon^{2}}\right]+\frac{K}{4}\left[\nabla \mathbf{n}:(\nabla \mathbf{n})^{T}+\frac{\left(|\mathbf{n}|^{2}-1\right)^{2}}{2 \delta^{2}}\right]+\mu_{\text {anch }},
$$


where

$$
\mu_{\text {anch }}= \begin{cases}-A \nabla \cdot[(\mathbf{n} \cdot \nabla \phi) \mathbf{n}] & \text { (planar anchoring) } \\ -A \nabla \cdot\left[|\mathbf{n}|^{2} \nabla \phi-(\mathbf{n} \cdot \nabla \phi) \mathbf{n}\right] & \text { (homeotropic anchoring) }\end{cases}
$$

We evolve the director field using the simplified Leslie-Ericksen theory of de Gennes and Prost [16], first used by Yue et al. [35], and in the twodimensional work of Mata et al. [26],

$$
\frac{\partial \mathbf{n}}{\partial t}=-\tau \frac{\delta F}{\delta \mathbf{n}}
$$

where $\tau$ is Leslie twist viscosity, which can be physically measured [16]. Then the coupled system of equations governing the phase separation of the mixture is

$$
\begin{aligned}
& \frac{1}{\gamma} \frac{\partial \phi}{\partial t}=\nabla^{2}\left[\lambda\left(-\nabla^{2} \phi+\frac{\phi^{3}-\phi}{\varepsilon^{2}}\right)\right.\left.+\frac{K}{4}\left(\nabla \mathbf{n}:(\nabla \mathbf{n})^{T}+\frac{\left(|\mathbf{n}|^{2}-1\right)^{2}}{2 \delta^{2}}\right)+\mu_{\text {anch }}\right] \\
& \frac{1}{\tau} \frac{\partial \mathbf{n}}{\partial t}=K\left[\nabla \cdot\left(\frac{1+\phi}{2} \nabla \mathbf{n}\right)-\frac{1+\phi}{2} \frac{\left(|\mathbf{n}|^{2}-1\right) \mathbf{n}}{\delta^{2}}\right]-\mathbf{g}_{\text {anch }},
\end{aligned}
$$

where $\mu_{\text {anch }}$ is given by (9) and

$$
\mathbf{g}_{\text {anch }}= \begin{cases}A(\mathbf{n} \cdot \nabla \phi) \nabla \phi & \text { (planar anchoring), } \\ A\left[|\nabla \phi|^{2} \mathbf{n}-(\mathbf{n} \cdot \nabla \phi) \nabla \phi\right] & \text { (homeotropic anchoring). }\end{cases}
$$

We non-dimensionalize the system (9)-(12) by selecting characteristic time, length, and energy scales $t_{c}, L_{c}$, and $E_{c}$, respectively. Then, the free energy parameters $K, A$, and $\lambda$ are made dimensionless with $E_{c} / L_{c}, \gamma$ with $L_{c}^{5} /\left(E_{c} t_{c}\right)$, and $\tau$ with $L_{c}^{3} /\left(E_{c} t_{c}\right)$. We choose the characteristic length scale $L_{c}=L / 2$, i.e. one half the domain size. Denoting by $K_{c}$ and $\tau_{c}$ characteristic values of the Frank elastic constant and the Leslie twist viscosity, respectively, we define characteristic energy and time scales by $E_{c}=a K_{c} L_{c}$, $t_{c}=b L_{c}^{3} /\left(E_{c} \tau_{c}\right)$, respectively, where $a$ and $b$ are dimensionless constants. Following $[26,35]$, we take $a=1 /\left(6.708 \times 10^{-3}\right)$ and $b=1$. We use the same letters to denote the dimensionless variables and parameters, so (9)-(12) can be considered to be in dimensionless form. We consider here only periodic boundary conditions. 


\section{Numerical Methodology}

We employ the same discretization as in [26], except that here we write the Cahn-Hilliard equation as a second order system to avoid a direct discretization of the fourth order, biharmonic operator and to use an efficient linear multigrid method $[13,12]$. The spatial discretization is second order with standard finite differences and periodic boundary conditions. The time integration is a linearly implicit scheme, as the one considered in $[1,12]$, in which the implicit part is discretized using a second-order backward difference formula (BDF) and the explicit part corresponds to a second order Adams-Bashforth method. The scheme can be written as

$$
\begin{aligned}
\frac{\frac{3}{2} \phi_{1}^{n+1}-2 \phi_{1}^{n}+\frac{1}{2} \phi_{1}^{n-1}}{\Delta t} & =\gamma \lambda \nabla^{2} \phi_{2}^{n+1}+2 \mathcal{F}^{n}-\mathcal{F}^{n-1}, \\
\phi_{2}^{n+1} & =\frac{\alpha}{\varepsilon^{2}} \phi_{1}^{n+1}-\nabla^{2} \phi_{1}^{n+1}, \\
\frac{\frac{3}{2} \mathbf{n}^{n+1}-2 \mathbf{n}^{n}+\frac{1}{2} \mathbf{n}^{n-1}}{\Delta t} & =\tau K \beta \nabla^{2} \mathbf{n}^{n+1}+2 \mathcal{G}^{n}-\mathcal{G}^{n-1},
\end{aligned}
$$

where $\alpha$ and $\beta$ are numerical parameters to improve numerical stability (in this work we take $\alpha=2$ and $\beta=1$ ), and $\mathcal{F}^{k}$ and $\mathcal{G}^{k}$, for $k=n-1, n$, are defined by the functions

$$
\begin{aligned}
& \mathcal{F}=\gamma \nabla^{2}[\left.\frac{\lambda}{\varepsilon^{2}}\left(\phi_{1}^{3}-\phi_{1}\right)+\frac{K}{4}\left(\nabla \mathbf{n}:(\nabla \mathbf{n})^{T}+\frac{\left(|\mathbf{n}|^{2}-1\right)^{2}}{2 \delta^{2}}\right)+\mu_{\text {anch }}\right] \\
& \quad-\gamma \lambda \frac{\alpha}{\varepsilon^{2}}\left(\frac{\alpha}{\varepsilon^{2}} \phi_{1}-\phi_{2}\right), \\
& \mathcal{G}=\tau K\left[\nabla \cdot\left(\frac{1+\phi_{1}}{2} \nabla \mathbf{n}\right)-\frac{1+\phi_{1}}{2} \frac{\left(|\mathbf{n}|^{2}-1\right) \mathbf{n}}{\delta^{2}}\right]-\tau K \beta \nabla^{2} \mathbf{n}-\tau \mathbf{g}_{\text {anch }},
\end{aligned}
$$

evaluated at $t_{k}$ for $k=n-1, n$.

\subsection{Accuracy and convergence test}

We performed an accuracy and convergence test of our numerical approach by using the techniques of manufactured solutions. That is, starting with smooth periodic functions, $\phi_{e}$ and $\mathbf{n}_{e}$ (with $\left|\mathbf{n}_{e}\right|=1$ ), we added a forcing term to equations (10) and (11) so that $\left(\phi_{e}, \mathbf{n}_{e}\right)$ becomes an exact solution of the so modified system. Using this exact solution, the error of the numerical approximation and the convergence rate was evaluated. The test confirmed convergence at a second order rate for both the phase field and the director. The details are provided in the Appendix. 


\section{Numerical Results}

We consider two cases of spinodal decomposition, one giving rise to bicontinuous structures and one in which the isotropic fluid component is nucleated in a nematic continuous phase.

\subsection{Parameters}

The dimensionless model parameters are chosen following [26, 35]. Specifically, we take $\lambda=1.342 \times 10^{-2}, \gamma=4 \times 10^{-5}, \delta=6.25 \times 10^{-2}, \tau=1$, $K=A=6.708 \times 10^{-3}$, and $\varepsilon=4 / 256$. Using that characteristic values of the elastic constant are $O\left(10^{-11}\right) N[16]$ and that surface anchoring strength is in the range $10^{-3}-10^{-6} \mathrm{~J} / \mathrm{m}^{2}$ we obtain a characteristic length in the range of $10^{-5}-10^{-8} \mathrm{~m}$ and the ratio of elastic to surface anchoring falls within bounds of known nematics, as discussed in [26].

The system of equations (9)-(12) is solved on the cube $[0,2] \times[0,2] \times[0,2]$, with periodic boundary conditions, and using a uniform grid of mesh size $h=2 / 256$. The time step is $\Delta t=0.1$ and remains constant throughout all the simulations. To limit the terms $(1+\phi) / 2$ from exceeding 1 due to numerical overshoot, we approximate this term by $(1+s \phi) / 2$, where $s=0.90$.

The free energy decreased monotonically per time step and the mean of $\phi$ was preserved accurately (with less than a 0.038 variation, at the end of $2.4 \times 10^{5}$ time steps) for all the simulations reported here.

\subsection{Bicontinuous Coarsening}

We consider an initial state defined by a small perturbation of the uniform, symmetric mixture

$$
\phi_{0}\left(x_{i}, y_{j}, z_{k}\right)=0+\xi_{i j k},
$$

where $\xi_{i j k}$ is a uniformly distributed random number in $(-\varepsilon, \varepsilon)$ for each grid index $(i, j, k)$. The parameter $\varepsilon$ is the same as that in the mixing energy (2), i.e., the dimensionless capillary length. The initial director field is given by

$$
\mathbf{n}_{0}\left(x_{i}, y_{j}, z_{k}\right)=\frac{\left(1,1, \omega_{i j k}\right)}{\sqrt{2+\omega_{i j k}^{2}}}
$$

where $\omega_{i j k}$ is a uniformly distributed random number in $(-0.05,0.05)$ for each grid index $(i, j, k)$. We computed the solution up to $t=24000$, when it 
is approaching steady state. For such a long time computation this required taking an enormous number of time steps equal to $2.4 \times 10^{5}$.

We consider first planar anchoring conditions. After a fast, $t=O(\varepsilon)$, transient domains rich in each of the mixture components begin to form and coarsen. This coarsening process is illustrated in Fig. 1, which shows snapshots of the phase field and the corresponding interfacial surface $(\phi=0)$ between nematic and isotropic fluid domains, at three well-separated times. Also shown in Fig. 1 (right) is a subsample of the director field. When the domain size is much larger than $K / W \approx \varepsilon$, there is strong anchoring and the director field becomes tangential to the interfacial surface. This strong anchoring induces a long range orientational distortion in the director field and creates a marked anisotropy in the system. This has a profound effect in the domain morphology selection, as pointed out by Mata et al. [26] for the two-dimensional system. In 3D, the mixture undergoes a more spectacular phase separation, as Fig. 1 shows; instead of the evolution toward lamellae observed in 2D [26] for either type of anchoring, the three-dimensional system passes from domains with a gyroid-like surface $[(c)(d)]$ to a structure whose isosurface has one pair of holes along each axis $[(\mathrm{e})(\mathrm{f})]$. At steady state, $t \approx 24000$, this domain interface seems to correspond to a notable minimal surface, the Schwarz P Surface, as Fig. 2 confirms.

We now look at the case of homeotropic anchoring, that is, when the favored orientation of the director field at a nematic-isotropic fluid interface (for domains much larger that $K / W$ ) is normal to that surface. The initial conditions are the same as before. Figure 3 depicts the coarsening dynamics in the presence of homeotropic anchoring. The plots correspond to the same snapshots $(t=600,4850,24000)$ as those of the planar anchoring case (Fig. 1). There is a striking difference in the morphology of the coarsening domains for the two types of anchoring. The preferential normal orientation of the director field at the coarsening domain interfaces gives rise to the selection of vertical lamellae, just as in the two-dimensional case [26].

The marked differences in morphology selection of the coarsening dynamics is highlighted in Fig. 4, which shows a comparison the phase separation for the two cases of anchoring with a spinodal decomposition of an isotropic mixture (modeled with the standard Cahn-Hilliard equation, i.e. $K=A=0$ ) at a late stage of the coarsening. The same initial conditions were used for the three cases. The emergence of vertical lamellae is evident in the isotropic, plain Cahn-Hilliard case but the orientation of the $\phi=0$ isosurfaces is quite different from the case with homeotropic anchoring. 


\subsection{Nucleation of an Isotropic Fluid in a Liquid Crystal Continuous Phase}

We now consider the case of asymmetric phase separation where the dominant component is the nematic liquid crystal phase. The initial condition for the phase field is

$$
\phi_{0}\left(x_{i}, y_{j}, z_{k}\right)=0.5+\xi_{i j k},
$$

where $\xi_{i j k}$ is a uniformly distributed random number in $(-\varepsilon, \varepsilon)$ for each grid index $(i, j, k)$. We take the initial director field as in (19). For this type of spinodal decomposition, small droplets of the isotropic fluid phase will nucleate in a liquid crystalline continuous phase and an (inverted nematic) emulsion will form [29]. Colloidal dispersions and emulsions of this type have attracted considerable attention because the orientational elasticity of the host fluid and the potential formation of topological defects can enable a fine degree of control of colloidal ordering and its stability [29, 24, 32, 31]. Here, we consider only homeotropic anchoring, as this is the prevalent, preferential orientation of the director field at the experimentally observed surfaces of the nucleated droplets [24].

We present three phase field snapshots, the corresponding isosurfaces, and a sampling of the director field in Fig. 5. Once the nucleated droplets achieve a diameter significantly larger than the extrapolation length $K / W$, which for our choice of parameters is approximately equal to the capillary length $\varepsilon$, the director field preserves a normal orientation at the surface of the droplets. It should be noted that this simple, phenomenological model do not support the $( \pm 1 / 2)$ topological defects that are believed to significantly limit coalescence and yield stable configurations of linear (chain) droplet aggregates [24]. Instead, the nucleated droplets eventually merge to form a single drop at steady state, as Fig. 5(e)(f) shows.

\subsection{Coarsening Rate}

The coarsening rate of phase domains in spinodal decomposition is usually estimated by looking at the time growth of the first moment of the structure function. This statistical approach requires many realizations of each type of spinodal decomposition to get accurate estimates of a characteristic length scale $L(t)$ of the phase domains. Unfortunately, this is computationally prohibitive for our current three dimensional system. We opt here to estimate the asymptotic coarsening rate by using the rate of decay of the 
mixing energy $[23,15]$. The argument is that, in the zero capillary length limit $\varepsilon \rightarrow 0$,

$$
E_{\text {mix }}=\frac{1}{V} \int_{\Omega} \frac{\lambda}{2}\left[|\nabla \phi|^{2}+\frac{\left(\phi^{2}-1\right)^{2}}{2 \varepsilon^{2}}\right] d x,
$$

where $V$ is the volume of $\Omega$, scales like $1 / L(t)$ [23].

The assumption is that $L(t)$ grows like $t^{\alpha}$, and we write $L(t) \sim t^{\alpha}$. Scaling arguments show that $\alpha=1 / 3$ for the constant diffusion Cahn-Hilliard equation with a Ginzburg-Landau free energy [6]. The two-dimensional study of Mata et al. [26] indicates that the growth rate $\alpha$ is affected by the anchoring conditions at the domain boundaries of a nematic liquid crystal-isotropic fluid mixture.

Figure 6 shows the striking effect of anchoring in the decay rate of $\log \left(E_{\text {mix }}\right)$, and consequently on the coarsening rate $\alpha$, for bicontinuous (symmetric) phase separation. With planar anchoring, there is a good single fit for a coarsening rate $\alpha$ which is clearly smaller than that of an isotropic binary mixture (phase separation governed by the Cahn-Hilliard equation). The orientational distortion in the nematic-rich phase, induced by the planar anchoring, slows down the coarsening of the separating mixture. This is consistent with what has been reported in the two-dimensional case [26]. However, the estimated exponent rate with planar anchoring in $3 \mathrm{D}(\alpha=0.2839)$ is appreciably larger than the corresponding in 2D $(\alpha=0.2317)$. But the more dramatic effects of the orientational distortion in the nematic phase on the coarsening dynamics occur with homeotropic anchoring, as Fig. 6 (b)indicates. There are three late coarsening stages with markedly different rates. In the first one, the domain coarsen at a rate $(\alpha=0.3204)$ commensurate with that of an isotropic, binary mixture. Then, after larger domains with highly curved boundaries have formed, there is a pronounced slow down in the coarsening $(\alpha=0.1886)$, which persists for a significant $t$ span, approximately from $t=2980$ to $t=8100$. This is then followed by an astonishing acceleration of the coarsening dynamics $(\alpha=0.6007)$ toward a steady state. While in $2 \mathrm{D}$ a larger than $1 / 3$ coarsening rate $(\alpha=0.4006)$ was also estimated for homeotropic anchoring in a symmetric mixture [26], the coarsening dynamics of the three-dimensional system is clearly different from its 2D counterpart.

To learn more about the three distinctive coarsening stages produced with homeotropic anchoring we present in Fig. 7 isosurface plots and a sample of the director field for representative times in each of the three stages. The coarsening regime with $\alpha=0.3204$, Fig. 7 (a), corresponds to the early stages 
of domain formation. The domain size has not reached the critical (extrapolation) length to fix the anchoring. As a result, the orientational field is largely isotropic and hence the coarsening rate is close to that of a binary mixture of isotropic fluids. Once the nematic-rich domains reach a critical size, there is strong anchoring which produces long range distortions of the orientational field, as Fig. 7(a) illustrates. The large elastic energy associated with these distortions leads to a significant slow-down of the domain coarsening. As the isosurfaces straighten up, Fig. 7(c), and the orientational field becomes largely uniform, a fast dynamics takes places leading to a lamellar steady state [Fig. 3(e)-(f)].

Finally, Fig. 8 shows the $\log \left(E_{m i x}\right)$ for nucleation coarsening of an isotropic fluid in a nematic liquid crystal continuous phase, with homeotropic anchoring. The initial conditions and parameters are as described in Subsection 4.3. In contrast with the late coarsening behavior of bicontinuous phase separation with the same anchoring [Fig. $6(\mathrm{~b})]$, the $\log \left(E_{m i x}\right)$ is well approximated by a linear fit, despite the presence of two small amplitude bumps near end. The corresponding coarsening rate $(\alpha=0.3423)$ is higher but very close to the isotropic (Cahn-Hilliard) coarsening rate. This contrasting coarsening behavior underlines the relevance of anchoring and free surface geometry.

\section{Concluding Remarks}

We considered a conserved phase field model to investigate numerically the three-dimensional coarsening dynamics of a nematic liquid crystal-isotropic fluid mixture. The model is a coupled system for a generalized Cahn-Hilliard equation for the phase order parameter $\phi$, related to the volume fraction of the nematic component, and a simplified de Gennes-Prost evolution equation for the director field $\mathbf{n}$, which describes the mean orientation of the rigid rod-like, liquid crystal molecules. Despite the evident limitations of this model (it is phenomenological, it describes the mean molecular orientation in terms of a vector field rather than using a second order, orientational tensor, the condition $\|\mathbf{n}\|=1$ is mollified, it does not capture disclinations, etc.), our study highlights the strong effects that anchoring of the nematic component on free surfaces can have on the coarsening rate and the steadystate morphology of phase separating mixture. It also shows that there are significant differences between the three-dimensional and two-dimensional coarsening processes. In particular, the study identifies a remarkable, new

3 -stage coarsening process with markedly different coarsening rates in the 
three-dimensional bicontinuous phase separation with homeotropic anchoring, unseen in the two-dimensional system.

Wall anchoring could have profound effects on the phase separation but was not considered here in order to focus the computational resources on freesurface anchoring; the computational cost to solve for steady or quasi-steady state is enormous. Each case took several months of CPU time of a stateof-the-art computer. We investigated the use of adaptive mesh refinements (AMR) $[13,12]$ as a means to reduce the overall computational cost per simulation. Unfortunately, the interfaces of the nematic liquid crystal and isotropic fluid domains occupy great part of the interior of the computational domain during most of the huge simulation time span, as Fig. 9 shows. This produces a high overhead due to large number of interpolations needed for the multi-level multi-grid linear solver and yields the AMR approach ineffective for this particular setting.

\section{Acknowledgements}

The authors gratefully acknowledge support by CAPES (RLN), the National Science Foundation Grant DMS 1317684 (HDC), and the program Ciências Sem Fronteiras, CNPq Brazil (HDC). RLN would like to acknowledge the hospitality of the Department of Mathematics at UCSB during his visit in which part of this research was conducted.

\section{Appendix: Accuracy and Convergence Test}

For this test we let

$$
\begin{aligned}
\phi_{e}(t, \mathbf{x}) & =\sin ^{3}(2 \pi(x+y+z+t)) \\
n_{x e}(t, \mathbf{x}) & =0 \\
n_{y_{e}}(t, \mathbf{x}) & =\cos (2 \pi(x+y+z+t)) \\
n_{z e}(t, \mathbf{x}) & =\sin (2 \pi(x+y+z+t))
\end{aligned}
$$

and the domain $\Omega$ be the unit cube. We modify (10) and (11) so that (22)(25) becomes an exact solution in $\Omega$ with periodic boundary conditions. We solve the modified system for $0<t \leq 10$, taking $\alpha=2, \beta=1, \varepsilon=0.125$, $\gamma=0.00001, \tau=0.5, \lambda=0.1, \delta=0.5$, and $K=A=0.006708$.

The numerical error (in the Euclidean norm) and the convergence ratio are presented in Table 1 for resolutions $\Delta t=h=1 / n$, with $n=32,64,128$, 
and 256. The observed convergence ratios confirm the expected second order accuracy (for smooth solutions) of the method for both the phase field $\phi$ and the director $\mathbf{n}$.

Table 1: Numerical error and convergence ratio at $t=10$ for resolution $\Delta t=h=1 / n$.

\begin{tabular}{|c|c|c|c|}
\hline$n$ & Variable & Error & Convergence ratio \\
\hline \hline 32 & $\phi$ & $\left\|\phi-\phi_{e}\right\|_{2}=2.269320008828050 \times 10^{-2}$ & \\
\hline & $n_{x}$ & $\left\|n_{x}-n_{x e}\right\|_{2}=1.649338034918546 \times 10^{-3}$ & \\
\hline & $n_{y}$ & $\left\|n_{y}-n_{y_{e}}\right\|_{2}=1.038634062541942 \times 10^{-2}$ & \\
\hline & $n_{z}$ & $\left\|n_{z}-n_{z e}\right\|_{2}=1.078656325408673 \times 10^{-2}$ & \\
\hline \hline 64 & $\phi$ & $\left\|\phi-\phi_{e}\right\|_{2}=6.059074243078967 \times 10^{-3}$ & 3.75 \\
\hline & $n_{x}$ & $\left\|n_{x}-n_{x e}\right\|_{2}=5.154721285378072 \times 10^{-4}$ & 3.20 \\
\hline & $n_{y}$ & $\left\|n_{y}-n_{y_{e}}\right\|_{2}=2.784391310165071 \times 10^{-3}$ & 3.73 \\
\hline & $n_{z}$ & $\left\|n_{z}-n_{z e}\right\|_{2}=2.659065270324107 \times 10^{-3}$ & 4.06 \\
\hline \hline 128 & $\phi$ & $\left\|\phi-\phi_{e}\right\|_{2}=1.545196350195382 \times 10^{-3}$ & 3.92 \\
\hline & $n_{x}$ & $\left\|n_{x}-n_{x e}\right\|_{2}=1.400611891955155 \times 10^{-4}$ & 3.68 \\
\hline & $n_{y}$ & $\left\|n_{y}-n_{y_{e}}\right\|_{2}=7.133143091353612 \times 10^{-4}$ & 3.90 \\
\hline & $n_{z}$ & $\left\|n_{z}-n_{z e}\right\|_{2}=6.613351900551691 \times 10^{-4}$ & 4.02 \\
\hline \hline 256 & $\phi$ & $\left\|\phi-\phi_{e}\right\|_{2}=3.885536109260787 \times 10^{-4}$ & 3.98 \\
\hline & $n_{x}$ & $\left\|n_{x}-n_{x e}\right\|_{2}=3.607710884265911 \times 10^{-5}$ & 3.88 \\
\hline & $n_{y}$ & $\left\|n_{y}-n_{y_{e}}\right\|_{2}=1.802250166619108 \times 10^{-4}$ & 3.96 \\
\hline & $n_{z}$ & $\left\|n_{z}-n_{z e}\right\|_{2}=1.652681029440823 \times 10^{-4}$ & 4.00 \\
\hline
\end{tabular}

\section{References}

[1] V. E. Badalassi, H. D. Ceniceros, and S. Banerjee. Computation of multiphase systems with phase field models. J. Comput. Phys., 190:371397, 2003.

[2] V. E. Badalassi, H. D. Ceniceros, and S. Banerjee. Gravitational effects on structural development in quenched complex fluids. Ann. NY. Acad. Sci., 1027:371-382, 2004. 
[3] I. Barosan, P.D. Anderson, and H.E.H. Meijer. Application of mortar elements to diffuse-interface methods. Computers \& Fluids, 35:13841399, 2006.

[4] F. Boyer. A theoretical and numerical model for the study of incompressible mixture flows. Computer \& Fluids, 31(1):41, 2002.

[5] F. Boyer, L. Chupin, and P. Fabrie. Numerical study of viscoelastic mixtures through a Cahn-Hilliard flow model. Euro. J. Mech. B /Fluids, 23:759-780, 2004.

[6] A. J. Bray. Theory of phase-ordering kinetics. Advances in Physics, 43(3):357-459, 1994.

[7] A. J. Bray. Coarsening dynamics of phase-separating systems. Phil. Trans. R. Soc. Lond. A, 361:781-792, 2004.

[8] S. Bronnikov, S Kostromin, and V.V. Zuev. Thermally induced isotropic-nematic phase separation in mixtures of low-molecular weight and polymer liquid crystals. Soft Materials, 11(1):6-12, 2013.

[9] S. Bronnikov, C. Racles, and V. Cozan. Kinetics of the nematic phase growth across the isotropic-nematic phase transition in polymerdispersed liquid crystals. Liquid Crystals, 36(3):319-328, 2009.

[10] J. W. Cahn and J. E. Hilliard. Free energy of a nonuniform system I. J. Chem. Phys., 28:258, 1958.

[11] J. W. Cahn and J. E. Hilliard. Free energy of a nonuniform system III. J. Chem. Phys., 31:688, 1959.

[12] H. D. Ceniceros, R. L. Nos, and A. M. Roma. Three-dimensional, fully adaptive simulations of phase-field fluid models. J. Comput. Phys., 229(17):6135-6155, 2010.

[13] H. D. Ceniceros and A. M. Roma. A nonstiff, adaptive, mesh refinementbased method for the Cahn-Hilliard equation. J. Comput. Phys., 225(2):1849-1862, 2007.

[14] R. Chella and V. Viñals. Mixing of a two-phase fluid by a cavity flow. Phys. Rev. E, 53:3832, 1996. 
[15] S. Dai and Q. Du. Computational studies of coarsening rates for the Cahn-Hilliard equation with phase-dependent diffusion mobility. $J$. Comput. Phys., 310:85-108, 2016.

[16] P. G. de Gennes and J. Prost. The Physics of Liquid Crystals. International Series of Monographs on Physics. Clarendon Press, 1993.

[17] H. Gomez and T. J. R. Hughes. Provably unconditionally stable, secondorder time-accurate, mixed variational methods for phase-field models. J. Comput. Phys., 230(13):5310-5327, 2011.

[18] P. C. Hohenberg and B. I. Halperin. Theory of dynamic critical phenomena. Rev. Mod. Phys., 49(3):435, 1977.

[19] D. Jacqmin. Calculation of two-phase Navier-Stokes flows using phasefield modeling. J. Comput. Phys., 155:96-127, 1999.

[20] Y. J. Jeon, Y. Bingzhu, J. T. Rhee, D. L. Cheung, and M. Jamil. Application and new developments in polymer-dispersed liquid crystal simulation studies. Macromolecular Theory and Simulations, 16(7):643-659, 2007.

[21] J. Kim, K. Kang, and J. Lowengrub. Conservative multigrid methods for Cahn-Hilliard fluids. J. Comput. Phys., 193:511-543, 2004.

[22] J. Kim, K. Kang, and J. Lowengrub. Conservative multigrid methods for ternary Cahn-Hilliard systems. Comm. Math. Sci., 2:53-77, 2004.

[23] R. V. Kohn and F. Otto. Upper bounds on coarsening rates. Commun. Math. Phys., 229:375-395, 2002.

[24] J.-C. Loudet, P. Barois, and P. Poulin. Colloidal ordering from phase separation in a liquid-crystalline continuous phase. Nature, 407:611-613, 2000 .

[25] J. Lowengrub and L. Truskinovsky. Quasi-incompressible Cahn-Hilliard fluids and topological transitions. Proc. R. Soc. London A, 454:2617, 1998.

[26] M. Mata, C. J. Garcia-Cervera, and H. D. Ceniceros. Ordering kinetics of a conserved binary mixture with a nematic liquid crytal component. Journal of Non-Newtonian Fluid Mechanics, 212:18-27, 2014. 
[27] A. Matsuyama and R. Hirashima. Phase separations in liquid crystalcolloid mixtures. The Journal of Chemical Physics, 128(4):044907, 2008.

[28] M. Motoyama, H. Nakazawa, T. Ohta, T. Fujisawa, H. Nakada, M. Hayashi, and M. Aizawa. Phase separation of liquid crystal-polymer mixtures. Computational and Theoretical Polymer Science, 10(3-4):287 $-297,2000$.

[29] P. Poulin, H. Stark, T.C. Lubenski, and D. A. Weitz. Novel colloidal interctions in anisotropic fluids. Science, 275:1770-1773, 1997.

[30] G. Sheng, T. Wang, Q. Du, K. G. Wang, Z. K. Liu, and L. Q. Chen. Coarsening kinetics of a two phase mixture with highly disparate diffusion mobility. Commun. Comput. Phys., 8(2):249-264, 2010.

[31] N.M. Silvestre, Q. Liu, B. Senyuk, I. Salyukh, and M. Tasinkevych. Towards template-assisted assembly of nematic colloids. Physical Review Letters, 112(22):225501, 2014.

[32] H. Stark. Physics of colloidal dispersions in nematic liquid crystals. Physics Reports, 351:387-474, 2001.

[33] O. Wodo and B. Ganapathysubramanian. Computationally efficient solution to the Cahn-Hilliard equation: Adaptive implicit time schemes, mesh sensitivity analysis and the 3D isoperimetric problem. J. Comput. Phys., 230(15):6037-6060, 2011.

[34] J. Xia, J. Wang, Z. Lin, F. Qiu, and Y. Yang. Phase separation kinetics of polymer dispersed liquid crystals confined between two parallel walls. Macromolecules, 39(6):2247-2253, 2006.

[35] P. Yue, J. J. Feng, C. Liu, and J. Shen. A diffuse-interface method for simulating two-phase flows of complex fluids. J. Fluid Mech., 515:293$317,2004$.

[36] P. Yue, J. J. Feng, C. Liu, and J. Shen. Diffuse-interface simulations of drop-coalescence and retraction in viscoelastic fluids. J. non-Newtonian Fluid Dynamics, 129:163-176, 2005.

[37] P. Yue, J. J. Feng, C. Liu, and J. Shen. Viscoelastic effects on drop deformation in steady shear. J. Fluid Mech., 540:427-437, 2005. 
[38] P. Yue, C. Zhou, J. J. Feng, C. F. Ollivier-Gooch, and H. H. Hu. Phasefield simulations of interfacial dynamics in viscoelastic fluids using finite elements with adaptive meshing. J. Comput. Phys., 219:47-67, 2006.

[39] T. Zhang and Q. Wang. Cahn-Hilliard vs singular Cahn-Hilliard equations in phase field modeling. Commun. Comput. Phys., 7(2, SI):362382,2010 . 


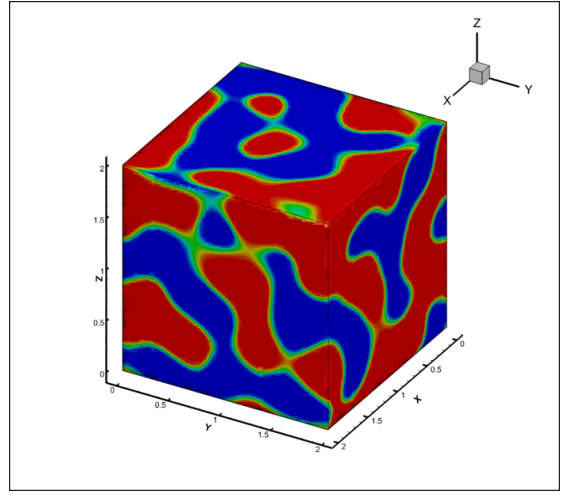

(a)

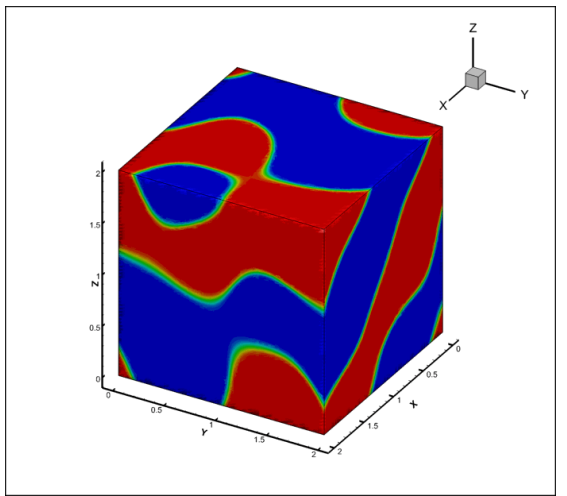

(c)

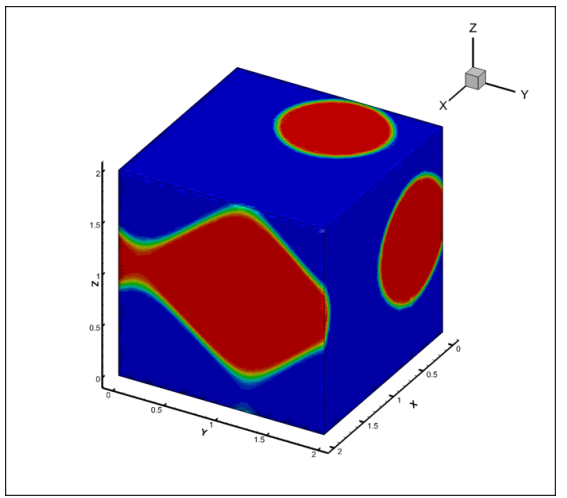

(e)

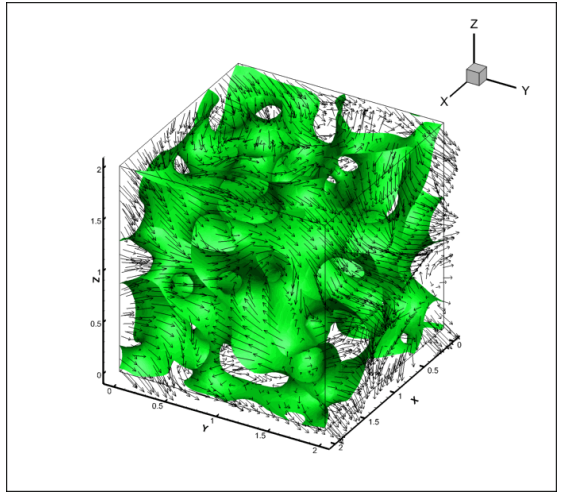

(b)

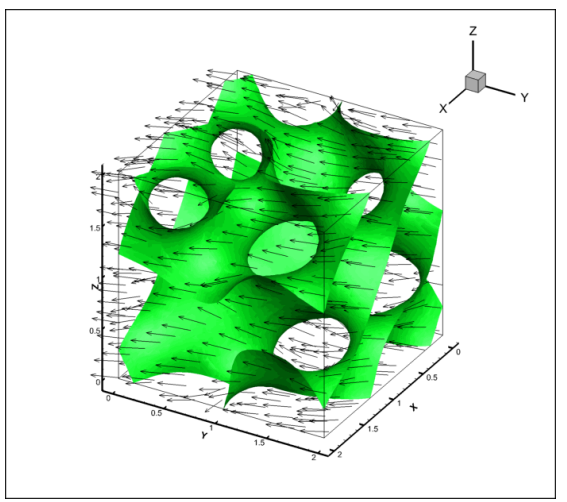

(d)

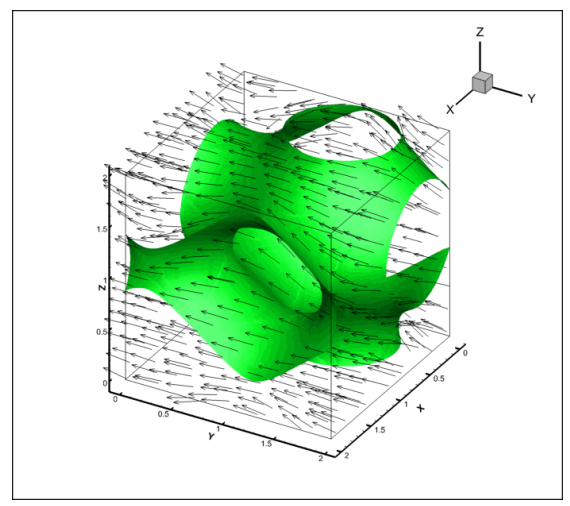

(f)

Figure 1: Bicontinuous coarsening with planar anchoring: phase field flooded contour plot (left) and isosurfaces $\phi=0$ with a sampling of the director field (right). (a)-(b) $t=600$, (c)-(d) $t=4850$, and (e)-(f) $t=24000$ (near steady state). Nematic phase in red (light) and isotropic fluid phase in blue (dark). 


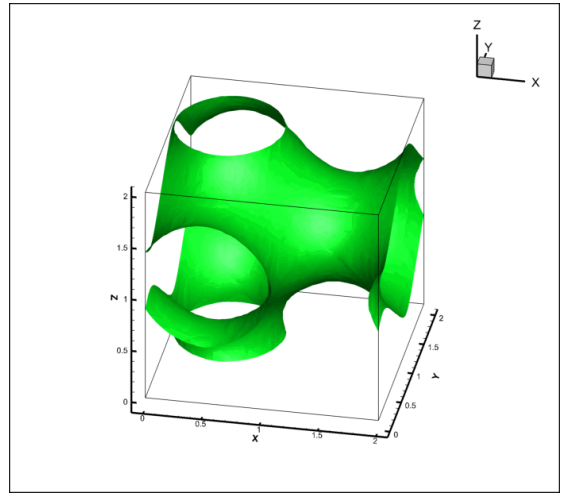

(a)

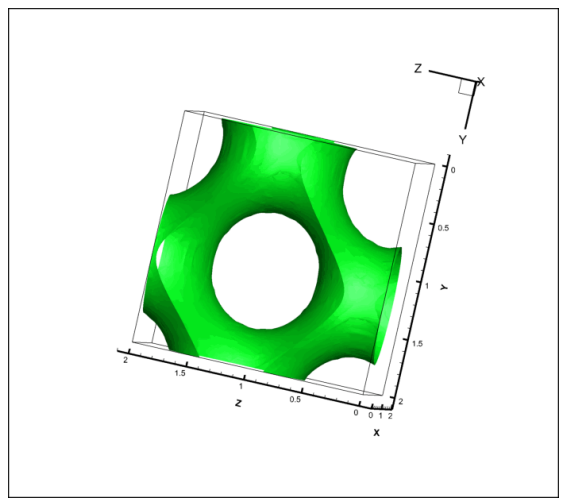

(b)

Figure 2: Schwarz P Surface. Rotated isosurface $\phi=0$, (a) and (b), for bicontinuous coarsening with planar anchoring at $t=24000$ (near steady state). 


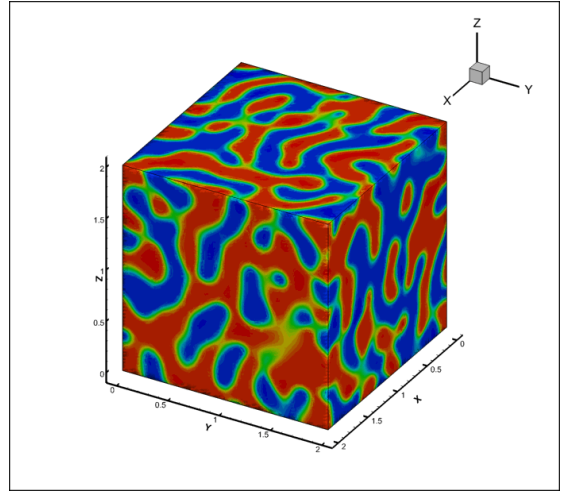

(a)

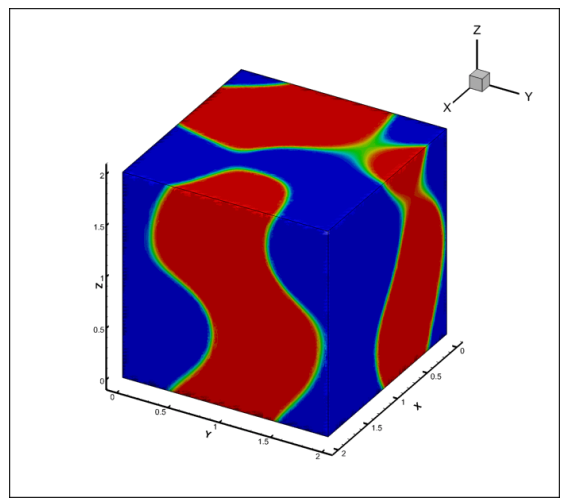

(c)

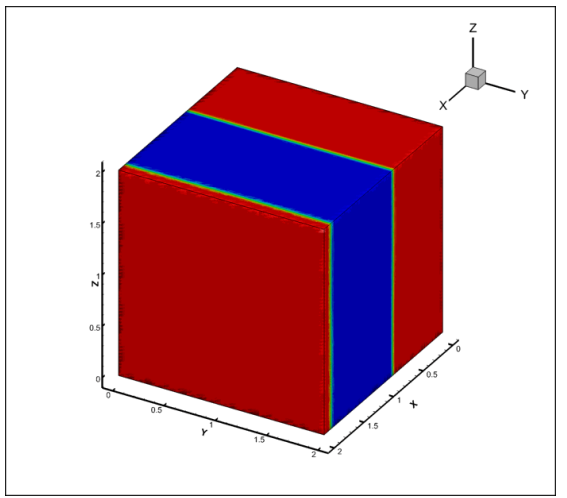

(e)

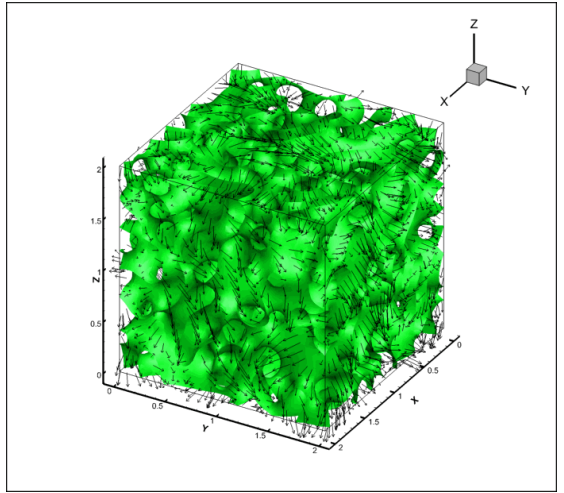

(b)

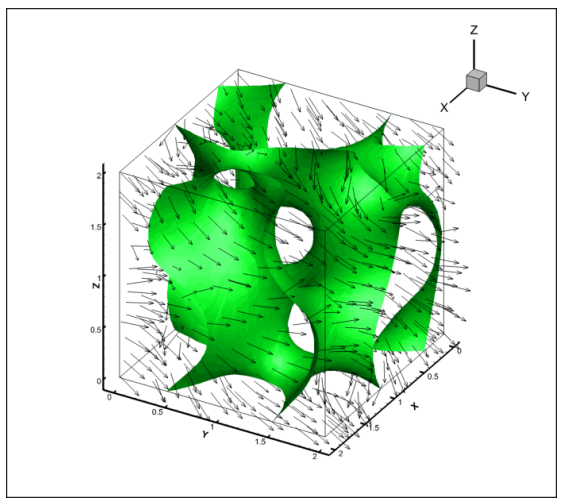

(d)

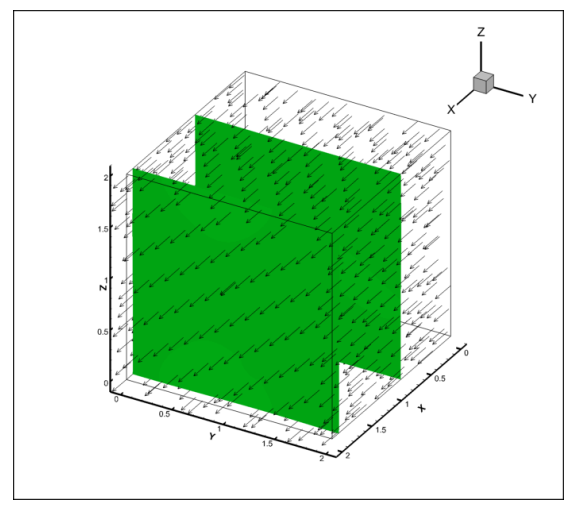

(f)

Figure 3: Bicontinuous coarsening with homeotropic anchoring: phase field flooded contour plot (left) and $\phi=0$ isosurfaces with a sampling of the director field (right). (a)-(b) $t=600$, (c)-(d) $t=4850$, and (e)-(f) $t=24000$. Nematic phase in red (light) and isotropic fluid phase in blue (dark). 


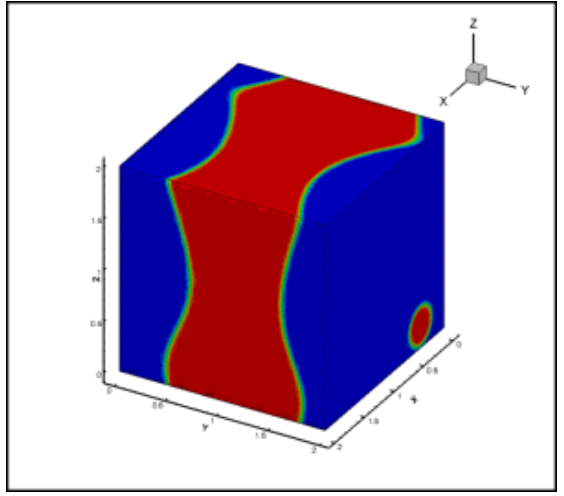

(a)

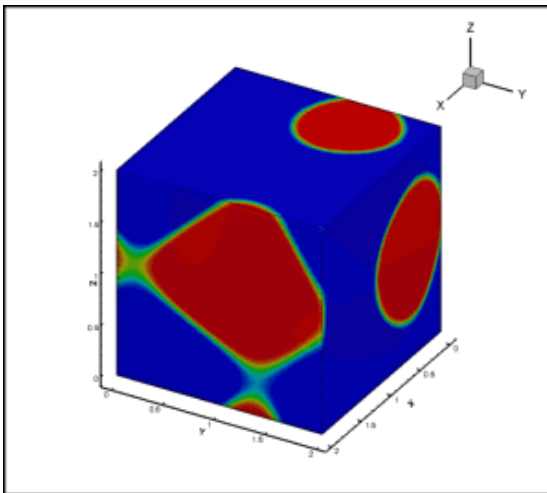

(c)

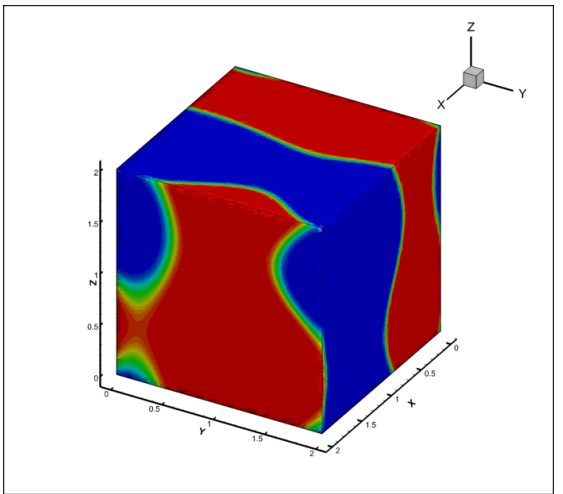

(e)

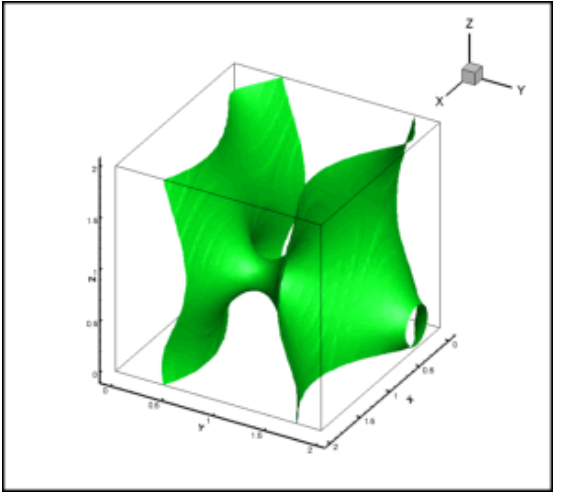

(b)

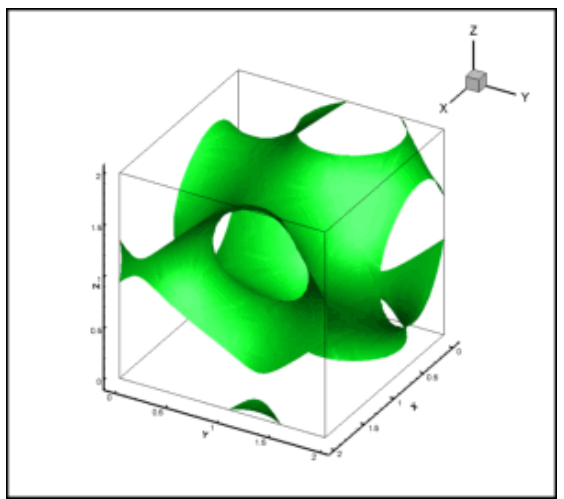

(d)

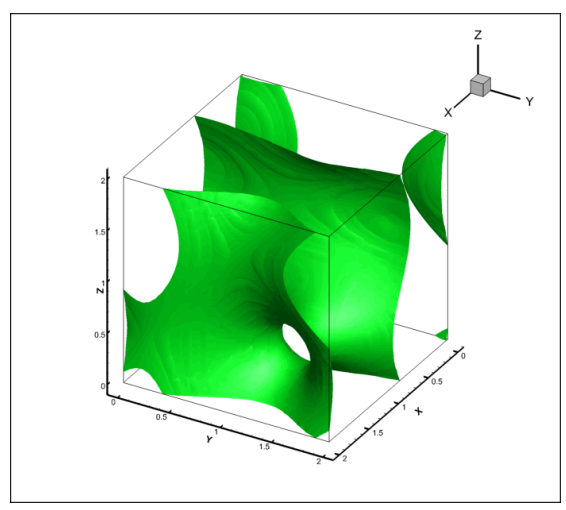

(f)

Figure 4: Comparison at $t=15000$ of the phase field (left) and the $\phi=0$ isosurface (right). (a) and (b) isotropic fluid mixture (Cahn-Hilliard), (c) and (d) nematic-isotropic fluid mixture with planar anchoring, and (e) and (f) nematic-isotropic fluid mixture homeotropic anchoring. Same initial condition for all cases. Nematic phase in red (light) and isotropic fluid phase in blue (dark). 


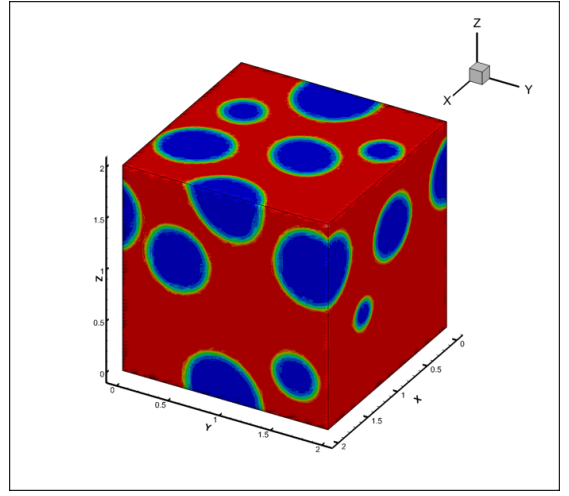

(a)

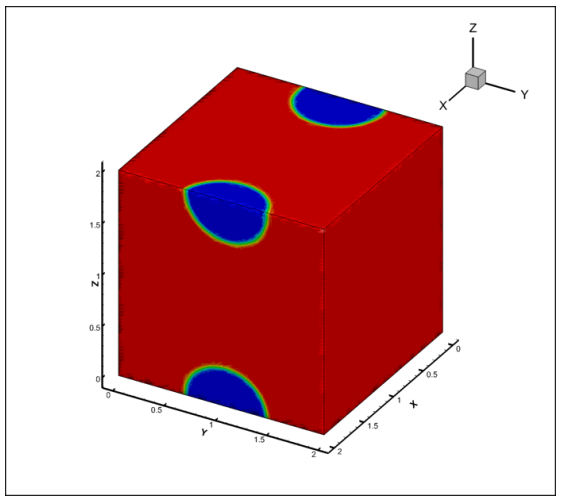

(c)

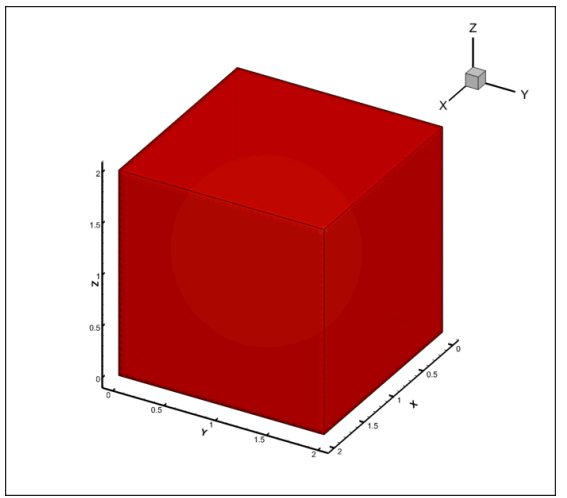

(e)

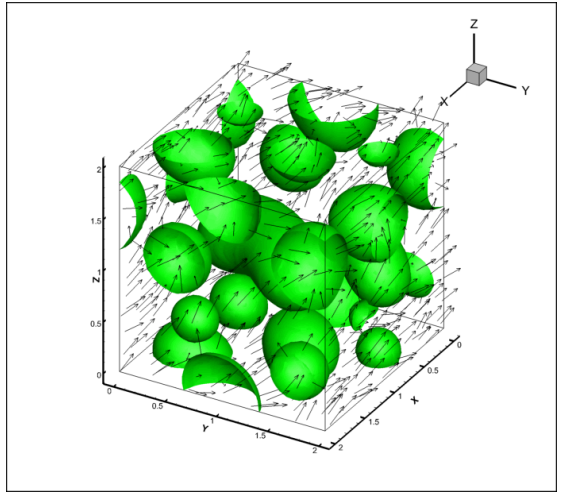

(b)

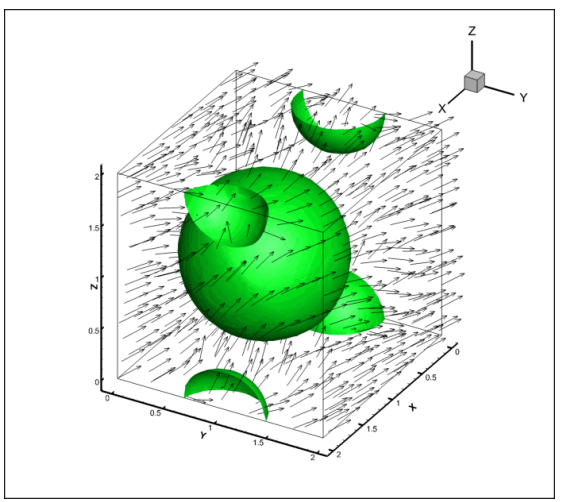

(d)

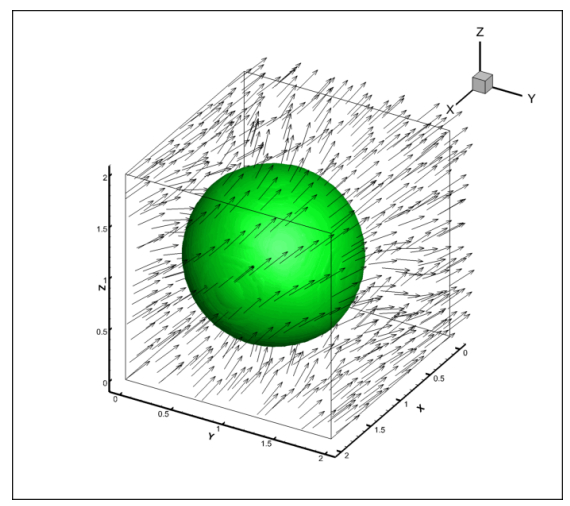

(f)

Figure 5: Nucleation coarsening with homeotropic anchoring: phase field flooded contour plot (left) and $\phi=0$ isosurfaces with a sampling of the director field (right). (a)-(b) $t=900$, (c)-(d) $t=7100$, and (e)-(f) $t=18000$. Nematic phase in red (light) and isotropic phase in blue (dark). 


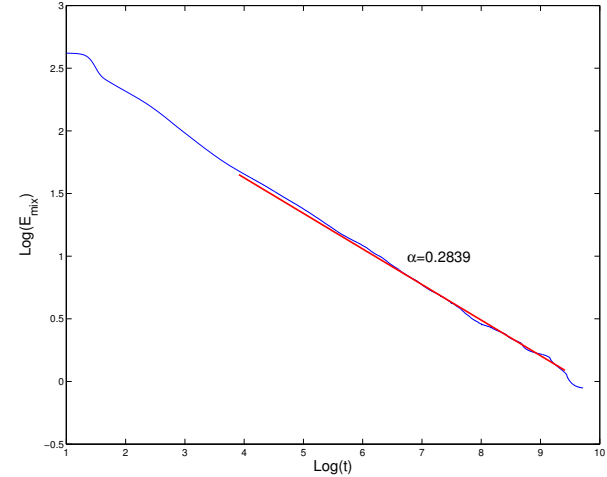

(a)

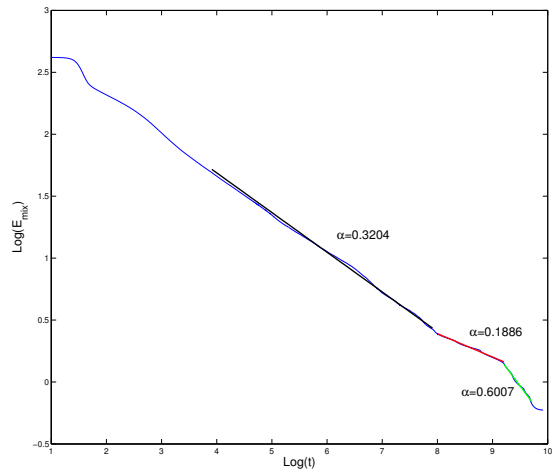

(b)

Figure 6: Logarithm of the mixing energy $E_{m i x}$ versus $\log (t)$ for bicontinuous coarsening (a) planar anchoring and (b) homeotropic anchoring. The constant $\alpha$ is the growth rate obtained by linear fitting of $\left(\log (t), \log \left(E_{m i x}\right)\right)$ in the regions marked with a straight line segment.

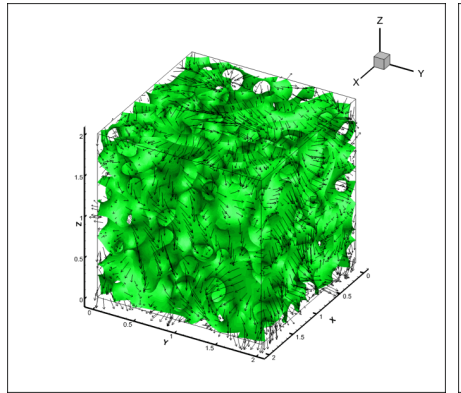

(a)

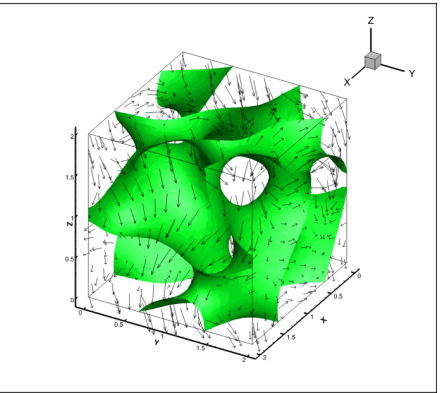

(b)

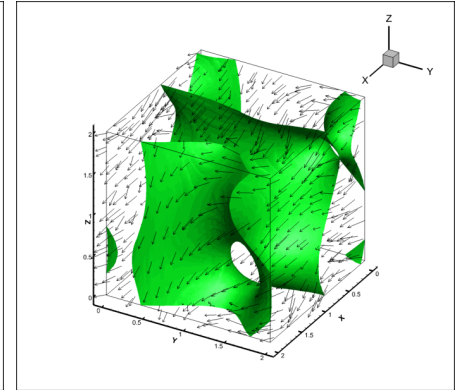

(c)

Figure 7: Isosurfaces and a sample of the director field for bicontinuous coarsening with homeotropic anchoring at the three identified coarsening regimes (a) $t=600$ ( $\alpha=0.3204$ stage), (b) $t=4700$ ( $\alpha=0.1886$ stage), and (c) $t=13800$ ( $\alpha=0.6007$ stage). 


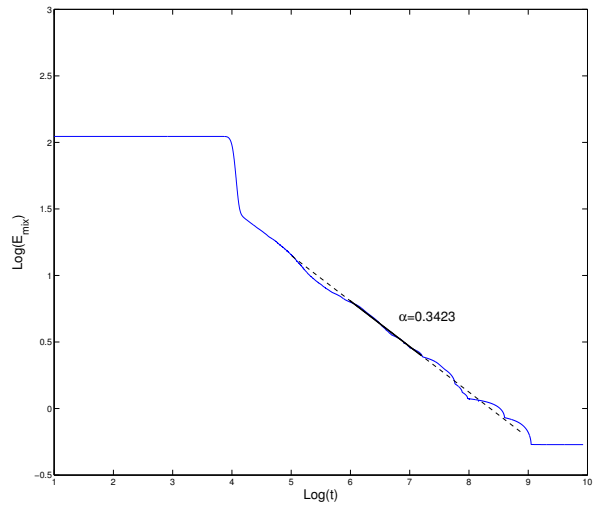

Figure 8: Logarithm of the mixing energy $E_{\text {mix }}$ versus $\log (t)$ for nucleation coarsening with homeotropic anchoring. The constant $\alpha$ is the growth rate obtained by linear fitting of $\left(\log (t), \log \left(E_{\text {mix }}\right)\right)$.

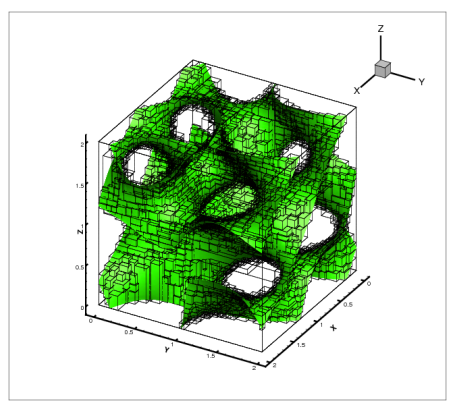

(a)

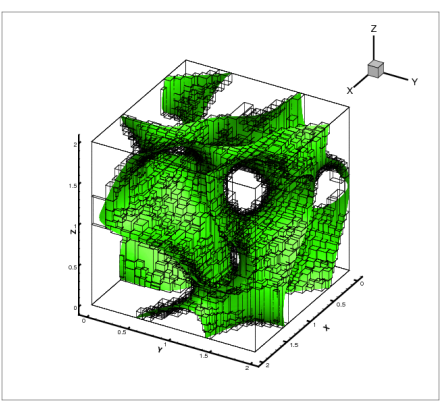

(b)

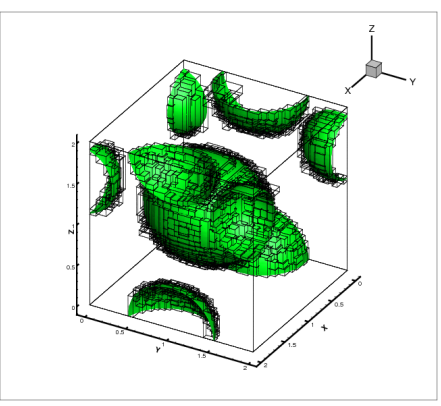

(c)

Figure 9: Isosurfaces $(\phi=0)$ and composite grid (a) planar anchoring at $t=4500$, (b) homeotropic anchoring at $t=5500$, and (c) nucleation with homeotropic anchoring at $t=3500$. 